\title{
HEART REDUCTION SURGERY: AN ANALYSIS OF THE IMPACT ON CARDIAC FUNCTION
}

Marc L. Dickstein, MD*

Henry M. Spotnitz, MD**

Eric A. Rose, $\mathrm{MD}^{* * *}$

Daniel Burkhoff, $\mathrm{MD}, \mathrm{PhD}^{* * * *}$
Objectives: Reports of improved ejection fraction, coupled with decreased filling pressures, have prompted a number of centers to begin evaluating the efficacy of heart reduction surgery to ameliorate symptoms of heart failure. However, the impact of this operation on cardiac mechanics is unknown. We applied a multiple compartment elastance model to simulate the effects of excising cardiac mass on heart function. Methods: The left ventricle was divided into two functional compartments to simulate excision of part of the wall. At multiple increments of mass reduction, the resulting end-systolic elastance, ejection fraction, stroke volume, end-diastolic pressure and volume, and diastolic stiffness were determined. Results: Changes in systolic function were accompanied by offsetting changes in diastolic function; consequently, overall pump function (the Frank-Starling Relationship) was found to be depressed. The geometric rearrangement associated with this operation leads to a reduction in wall stress for a given level of pressure generation, thus implying an increase in the efficiency with which wall stress is transduced into intraventricular pressure. Conclusions: Overall pump function is depressed in the short run after heart reduction surgery. However, on the basis of theoretic arguments, heart reduction surgery may have long-term beneficial implications. Importantly, this analysis revealed that changes in parameters of ventricular function have different implications during heart reduction surgery than when such changes are observed with inotropism caused by acute pharmacologic therapy. (J Thorac Cardiovasc Surg 1997;113:1032-40)
$\mathrm{H}$ eart reduction surgery has been recently introduced as a treatment for end-stage dilated cardiomyopathy. Considerable interest in this procedure has been generated by reports of clinical improvement in a number of patients after this operation. ${ }^{1}$ Reports of improved ejection fraction, coupled with decreased filling pressures, have prompted a number of centers to begin evaluating

From the College of Physicians and Surgeons, Columbia University, New York, N.Y.

Received for publication Sept. 23, 1996; revisions requested Nov. 4, 1996; revisions received Jan. 29, 1997; accepted for publication Jan. 29, 1997.

Address for reprints: Marc L. Dickstein, MD, Department of Anesthesiology, College of Physicians and Surgeons, Columbia-Presbyterian Medical Center, 630 West 168th St., New York, NY 10032.

*Department of Anesthesiology.

${ }^{* *}$ George H. Humphreys Professor of Surgery.

***Valentine Mott-Johnson \& Johnson Professor of Surgery.

****Division of Circulatory Physiology.

Copyright $\odot 1997$ by Mosby-Year Book, Inc.

$0022-5223 / 97 \$ 5.00+0 \quad \mathbf{1 2 / 1 / 8 0 7 7 6}$ the efficacy of heart reduction surgery to ameliorate symptoms of heart failure. However, little is known about the effects of this procedure on cardiac mechanics; moreover, the significance of changes in cardiac mechanics resulting from mass reduction on overall pump performance is unclear.

Ordinarily, evaluation of a therapy like heart reduction surgery would be facilitated through the use of experimental models. However, lack of an appropriate animal model may limit opportunities to obtain clinically relevant experimental data. Furthermore, in the clinical setting it is difficult to make the necessary measurements that are required for a full understanding of the impact of this procedure on cardiovascular function. As a consequence, interpretation of the available data may be limited. For example, while improvement in ejection fraction under normal circumstances would generally signify improvements in myocardial contractile state and pump function, this may not be the case under conditions of surgically altered ventricular mass.

Major advances have been made in understanding the relationship between intrinsic myocardial con- 
tractile properties, muscle mass, and global ventricular pump function. Through the use of pressurevolume and stress-strain analyses, it is possible to characterize and predict detailed aspects of cardiovascular dynamics under a variety of conditions. We therefore used these analytic tools to derive insights into the hemodynamic consequences of heart reduction surgery. Particular attention was given to predicting the effects on end-systolic and end-diastolic pressure-volume relations (ESPVRs and EDPVRs), stress-strain relations, ejection fraction, and overall pump performance (Frank-Starling relationship). The limitations and potential clinical implications are discussed.

\section{Methods and materials}

Overview of cardiovascular model. The cardiovascular system was modeled as described previously. ${ }^{2-4}$ In brief, the dynamics of right (RV) and left ventricular (LV) contraction were represented individually by modified nonlinear time-varying elastance models that interrelate instantaneous ventricular pressures and volumes:

$$
\mathrm{P}(\mathrm{V}, \mathrm{t})=\left(\mathrm{P}_{e s}[\mathrm{~V}]-\mathrm{P}_{e d}[\mathrm{~V}] \mathrm{e}[\mathrm{t}]\right)+\mathrm{P}_{e d}(\mathrm{~V})
$$

where $\mathrm{P}_{\mathrm{es}}(\mathrm{V})$ is a function that describes the ESPVR, $\mathrm{P}_{\mathrm{ed}}(\mathrm{V})$ is a function that describes the EDPVR, and $\mathrm{e}(\mathrm{t})$ is a function that describes the time course with which myocardial properties vary between end-diastole and endsystole. These functions are as follows ${ }^{2-4}$ :

$\mathrm{e}(\mathrm{t})=0.5\left(\sin \left[\pi \mathrm{t} / \mathrm{T}_{e s}-\pi / 2\right]+1\right)$

$$
\text { for } \mathrm{t}<3 \mathrm{~T}_{e s} / 2
$$

$\mathrm{e}(\mathrm{t})=0.5 \exp \left(\left[\mathrm{t}-3 \mathrm{~T}_{e s} / 2\right] / \mathrm{t}\right)$

$$
\begin{gathered}
\text { for } \mathrm{t} \leq 3 \mathrm{~T}_{e s} / 2 \\
\mathrm{P}_{e s}(\mathrm{~V})=\mathrm{E}_{e s}\left(\mathrm{~V}-\mathrm{V}_{o}\right) \\
\mathrm{P}_{e d}(\mathrm{~V})=\mathrm{A}\left(\exp \left[\mathrm{K}\left(\mathrm{V}-\mathrm{V}_{o}\right)\right]-1\right)
\end{gathered}
$$

where $T_{e s}$ is the time to end-systole, $E_{e s}$ is the end-systolic elastance, $V_{o}$ is the unstressed ventricular volume, $A$ is a scaling factor, and $\mathrm{K}$ is the diastolic stiffness coefficient. With this representation, ventricular chamber contractility is indexed by end-systolic elastance. ${ }^{5}$ The algorithm for coupling the ventricles to the systemic and pulmonary vascular systems is detailed in the appendix.

Heart reduction. To simulate mass reduction surgery, we made the following assumptions: the LV wall was of uniform thickness, muscle properties were homogeneous, and the chamber was rotationally symmetric; we also assumed that RV chamber properties were unaffected by the ventricular resection. The $\mathrm{LV}$ chamber was functionally divided into two compartments, one of which represents the portion to be removed (as shown schematically in Fig. 1). If the fraction of total LV volume contained within the compartment to be removed is defined as $r$, then the instantaneous relationship between pressure and
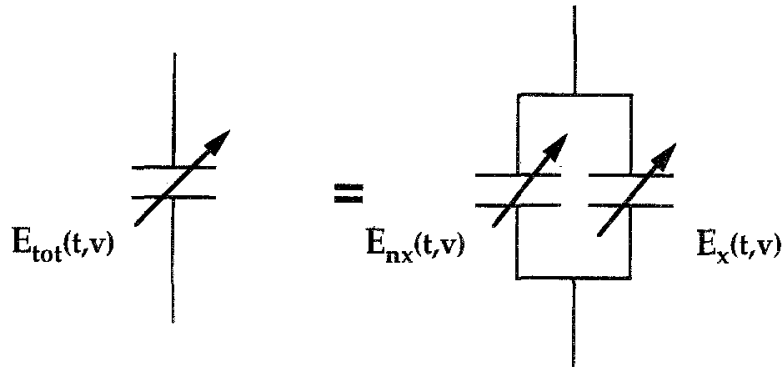

Fig. 1. To simulate mass reduction surgery, the total heart, represented by a modified nonlinear time-varying elastance $\left(E_{\text {tot }}[t, v]\right)$, was functionally divided into two portions: the portion to be excised $\left(E_{x}[t, v]\right)$ and the portion that is not excised $\left(E_{n x}[t, v]\right)$. Details of the computations are provided in the text (equations 1 to 6 ).

volume of the nonexcised compartment $\left(\mathrm{P}_{\mathrm{nx}}[\mathrm{V}, \mathrm{t}]\right)$ relates to equation 1 as follows:

$$
\mathrm{P}_{n x}(\mathrm{~V}, \mathrm{t})=\mathrm{P}(\mathrm{V} /[1-\mathrm{r}], \mathrm{t})
$$

The amount of muscle removed $\left(M_{x}\right)$ varies with $r$ as detailed in the appendix. Mass reduction was assumed to reduce LV chamber volume while leaving baseline wall thickness unchanged.

To ensure that the findings based on this multicompartmental model are not unique to this approach, we also performed a simple finite element analysis that allowed for prediction of ESPVR and EDPVR from specified elemental myocardial properties assuming a hemielliptic ventricular geometry. Details are presented in the appendix.

Protocol. The value of $\mathrm{r}$ was varied to achieve mass reductions of 25,50 , and $75 \mathrm{gm}$ of myocardium. At each value of $r$, we determined the resulting end-systolic elastance, ejection fraction, stroke volume, stroke work, LV end-diastolic pressure (LVEDP), the diastolic stiffness coefficient (K) of the EDPVR, and LV end-diastolic volume (LVEDV). Under each mass condition, hemodynamic data were also determined at several different preload conditions to examine the impact of mass reduction on LV pressure-volume relations, stroke workLVEDV relations (preload recruitable stroke work), stroke volume-LVEDP relations (Frank-Starling curves), and myocardial stress and strain. Thus, with the exception of LV mass and intravascular volume, all parameter values were maintained constant for the entire analysis.

\section{Results}

ESPVRs, EDPVRs, and their corresponding pressure-volume loops obtained in the baseline heart failure state and after the three degrees of mass reduction are shown in Fig. 2. This figure reveals that each increment of mass reduction was associated with a leftward shift in ESPVR and EDPVR; the magnitude of the volume-axis shifts were identical for both relations. These same shifts 


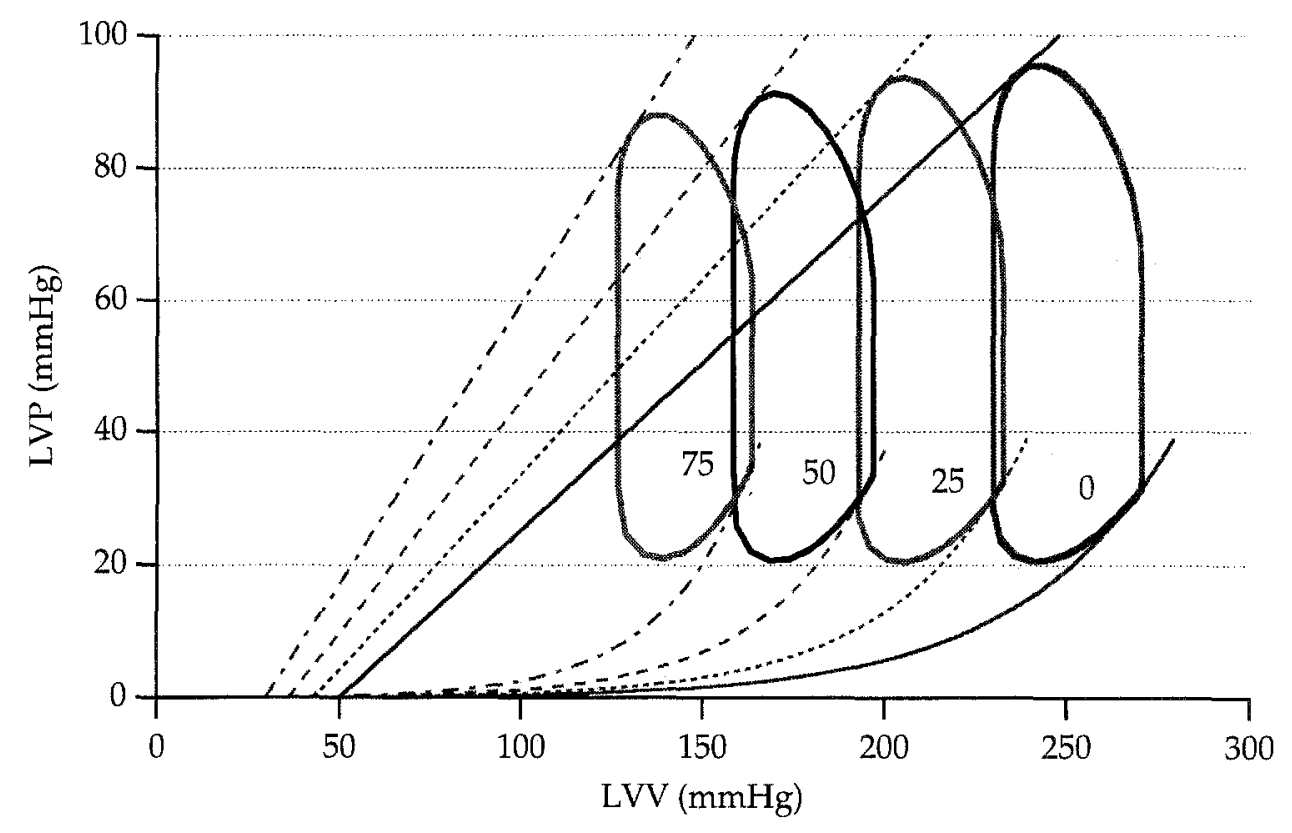

Fig. 2. Left ventricular pressure-volume loops $(L V P$ and $L V V)$ are shown at each of the four levels of mass reduction $(0,25,50$, and $75 \mathrm{gm}$ of tissue removed). The pressure-volume loops shift to the left in response to mass reduction. Also shown on this graph are the ESPVRs (straight lines that intersect the end-systolic point of the respective pressure-volume loop) and the EDPVRs (curvilinear relations that intersect the end-diastolic point of the respective pressure-volume loop). As mass is reduced from 0 to $75 \mathrm{gm}$ (solid line, dotted line, dashed line, dotted/dashed line), the ESPVR slope increases, and the curvature of the EDPVR increases; both relations tend to move in a parallel fashion.

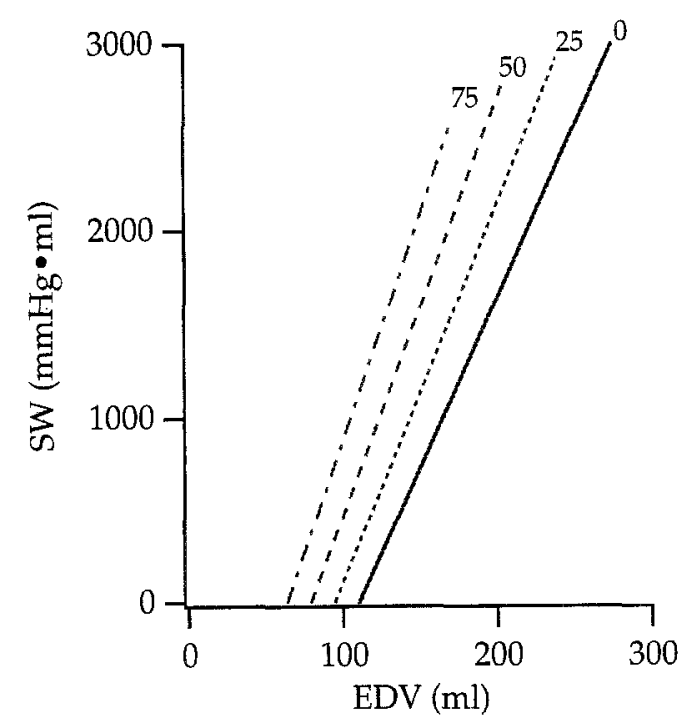

Fig. 3. Left ventricular stroke work-end-diastolic volume relations ( $S W$ and $E D V$ ). Similar to the ESPVR, the slope increases and volume-axis intercept decreases with incremental reductions in ventricular mass $(0 \mathrm{gm}$, solid line; 25 $\mathrm{gm}$, dotted line; $50 \mathrm{gm}$, dashed line; $75 \mathrm{gm}$, dotted/dashed line). in pressure-volume relations were also found with the finite-element analysis described in the appendix (Appendix Fig. 1). The pressure-volume loops shifted, correspondingly, with little change in filling pressure, stroke volume, peak systolic pressure, or overall loop shape. The stroke work-LVEDP relation derived from these loops is shown in Fig. 3 and an increase in slope in response to mass reduction reflecting increased systolic chamber function. The hemodynamic variables derived from these loops as a function of mass removed are shown in Fig. 4. Mass reduction caused increases in end-systolic elastance and ejection fraction (panel $A$ ), decreases in LVEDV (panel $B$ ), and increases in diastolic chamber stiffness $(K$, panel $B)$.

The effect of mass reduction on overall pump function was assessed by the relation between filling pressure and stroke volume, as shown in Fig. 5. These Frank-Starling curves, which exhibited the typical exponential rise to a plateau, moved downward and rightward in response to mass reduction indicating a slight reduction in pump performance.

To understand how mass reduction affects the 
A

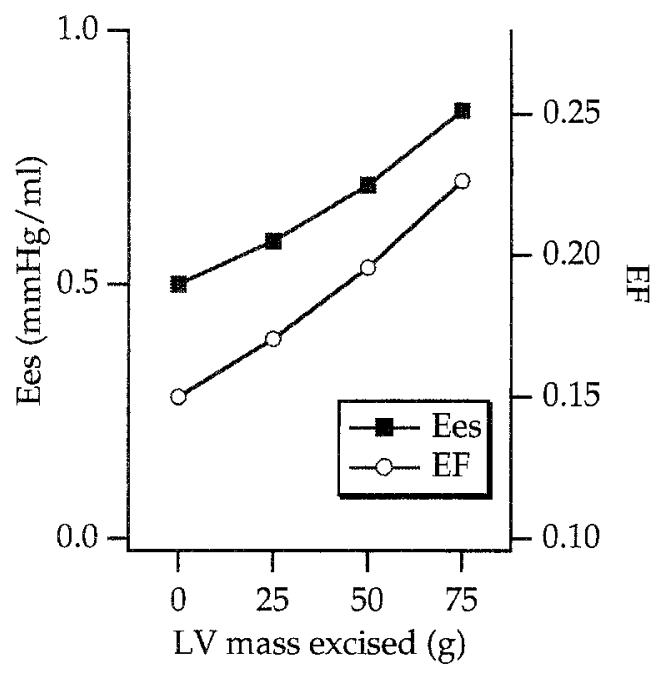

B

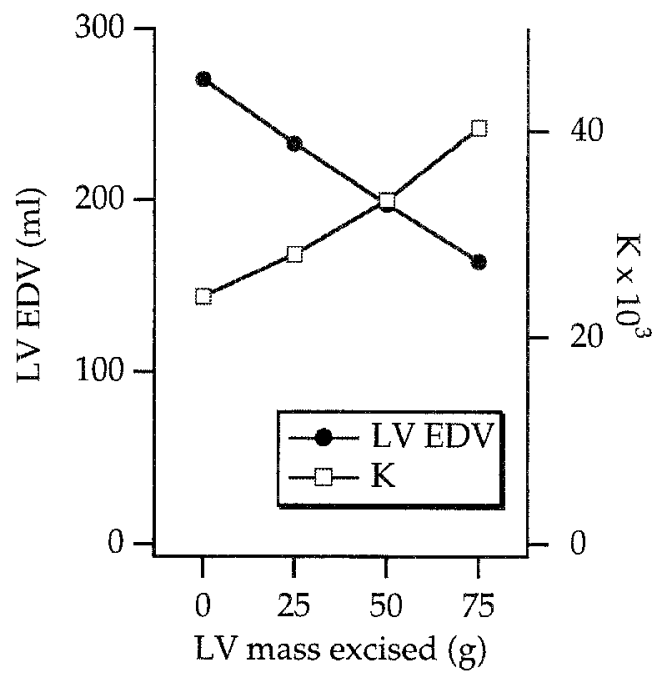

Fig. 4. A, Slope of the end-systolic pressure-volume relation (Ees; filled squares) and ejection fraction (EF; open circles) as a function of amount of LV mass excised ( $x$-axis). Both of these indices of systolic function increase with mass reduction. B, Left ventricular end-diastolic volume ( $L V E D V$; filled circles) and the stiffness coefficient of the end-diastolic pressure-volume relation ( $K$; open squares) also as a function of LV mass excised. Both of these measures reflect the decrease in chamber compliance associated with mass reduction.

mechanical stresses to which the myocardium is exposed, we converted the pressure-volume loops of Fig. 2 to stress-strain loops, as shown in Fig. 6. Myocardial stresses and strains ( $\sigma$ and $\epsilon$, respectively) were calculated according to the following standard equations ${ }^{6}$ :

$$
\begin{gathered}
\sigma=1.355 \mathrm{P}_{L V}\left(1+\left[3 \mathrm{~V}_{L V} / \mathrm{V}_{m}\right]\right) \\
\epsilon=1 / 3\left(\left[\operatorname{In}\left(1+\left[3 \mathrm{~V}_{L V} / \mathrm{V}_{m}\right]\right)\right]-\right. \\
\left.\left[\operatorname{In}\left(1+\left[3 \mathrm{~V}_{o} / \mathrm{V}_{m}\right]\right)\right]\right)
\end{gathered}
$$

where $P_{L V}$ and $V_{L V}$ are instantaneous LV pressure and volume, respectively, $V_{O}$ is the volume-axis intercept of the EDPVR, and $V_{m}$ is the volume of the myocardium (0.96 LV mass). With each reduction in mass, there was a reduction in peak stress along with an increase in loop width (increased shortening). These changes indicate that mass reduction is associated with a decrease in myocardial afterload stress.

To more closely examine the impact of mass reduction on myocardial afterload stress, we determined the relationship between peak pressure and peak stress over a range of preloading conditions (Fig. 7). At any ventricular pressure, mass reduction resulted in a decrease in ventricular stress. This analysis highlights the improved efficiency of transducing wall stress to chamber pressure after mass reduction.

\section{Discussion}

Although heart reduction surgery is now being performed at several centers, little is known about the impact of this procedure on either intrinsic heart function or overall cardiovascular hemodynamics. The potential for gaining further insights through experimental work is limited by the current lack of suitable animal models in which this procedure can be studied; furthermore, measurements that can typically be made in the clinical setting may provide only limited information about the underlying physiology. Accordingly, it would be beneficial to take advantage of any available techniques that may provide such insight.

Over the past 20 years, pressure-volume relationships and the time-varying elastance theory have evolved as relatively simple approaches to explaining the dynamics of ventricular contraction and ventricular-vascular interactions under a variety of physiologic and pathophysiologic conditions. ${ }^{7}$ Experience has shown that use of these approaches can prospectively lead to important understanding of 


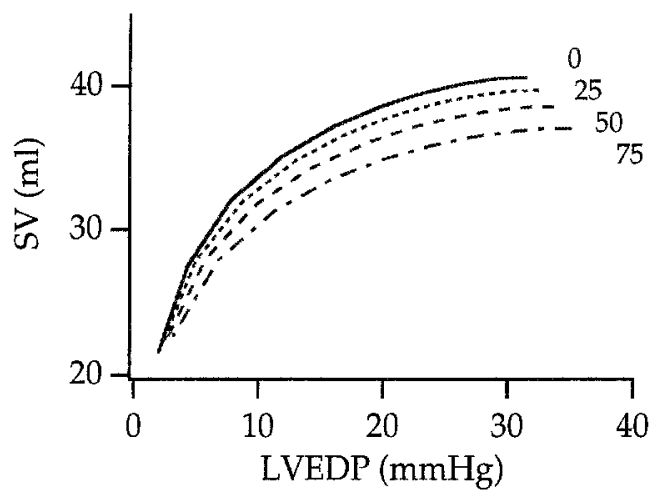

Fig. 5. Frank-Starling relationship relating stroke volume to LVEDP at the four mass reduction conditions ( 0 to 75 gm of tissue excision). Notice that mass reduction is associated with a downward shift of the Frank-Starling curves, indicating a reduction in pump function.

fundamental physiologic cardiovascular concepts. Specific examples that are pertinent to the present investigation are the successful use of so-called multicompartment models of the LV chamber (i.e., functionally dividing the heart into different compartments) to predict pump properties in the face of regional ischemia ${ }^{8}$ and abnormal activation sequences ${ }^{9,10}$ and to explain interventricular interactions. ${ }^{11,3}$ In a similar vein, we mimicked the situation of heart reduction surgery applied to hearts with idiopathic dilated cardiomyopathy by functionally dividing the entire ventricular elastance into a portion to be removed and a portion to be retained; although this approach has limitations (discussed below), our analysis has provided potentially important insights.

Reports of preliminary clinical experiences suggest that an early effect of mass reduction surgery is in improvement in ejection fraction. This phenomenon was readily predicted by the present analysis. In addition, there was an increase in end-systolic elastance and preload recruitable stroke work, load-independent indices of chamber contractile strength. Although these changes ordinarily signify significant improvements of ventricular performance, they may not have the same clinical or physiologic meaning after mass reduction as they do in other acute settings. For example, acutely improved indices of chamber contractility seen with $\beta$-agonist therapy normally signify an increase in myocardial contractility. In contrast, improvements in these indices after mass reduction are based on geometric rearrangement with no effect on underly-

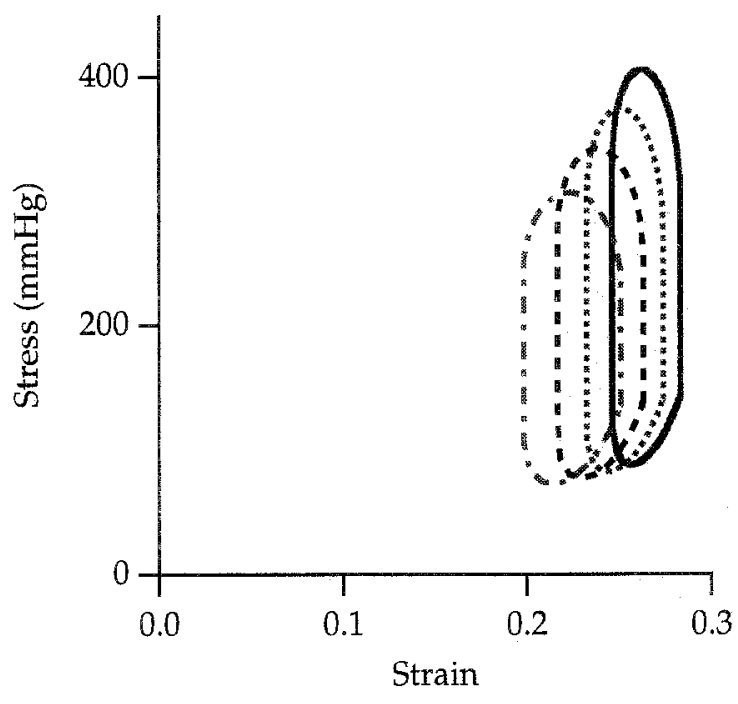

Fig. 6. Left ventricular stress-strain loops at each of the four levels of mass reduction ( $0 \mathrm{gm}$, solid line; $25 \mathrm{gm}$, dotted line; $50 \mathrm{gm}$, dashed line; $75 \mathrm{gm}$, dotted/dashed line). With mass reduction, there is a decrease in the peak stress attained, a small leftward shift of the relations, and a widening of the loops.

ing myocardial function. Furthermore, the improved ejection fraction was due to a decrease in enddiastolic volume with little change in stroke volume; the implications are different from those of a pharmacologically induced increase in ejection fraction, which is associated with increased stroke volume with relatively fixed end-diastolic volume. Finally, and also in sharp contrast to pharmacologically induced inotropism, improvements in systolic function indices seen with mass reduction are accompanied by counteracting changes in diastolic properties. As a result, indices of overall pump function such as provided by the Frank-Starling relationship are affected in a negative way by mass reduction; predicted shifts in this relationship, although small in magnitude, are comparable with those that would ordinarily be associated with a slight negative inotropic agent. Thus the balanced effect of mass reduction on systolic and diastolic properties confounds interpretation of an increased ejection fraction, end-systolic elastance, and preload recruitable stroke work; increases in these indices are usually associated with improved pump function, but this does not appear to be the case following mass reduction surgery.

In addition to these acute effects on global hemodynamics, mass reduction had a significant effect on the mechanical stress experienced by the myocar- 
dium. Specifically, for any given systolic pressure, a decrease in stress occurred. Consistent with the increase in ejection fraction, there was also an increase in myocardial shortening (i.e., in the change in strain during a beat) despite the relatively little change in stroke volume. Thus mass reduction causes acute myocardial afterload stress reduction by a mechanism that is fundamentally different from that normally seen with pharmacologic afterload reduction therapy. The latter is typically mediated by a reduction in arterial vascular resistance and is also associated with an increase in stroke volume and decrease in blood pressure. The implications of decreased afterload stress after mass reduction have several potentially important implications. First, myocardial hypertrophy in heart failure is generally believed to occur in response to the increased wall stress. ${ }^{12}$ Myocardial hypertrophy may of itself contribute to contractile dysfunction above that resulting from the underlying myopathy. ${ }^{13-15}$ Reduction of wall stress may therefore lead to regression of hypertrophy, which could potentially translate into a clinical benefit. However, such an effect could not be acute, since regression of hypertrophy would occur over weeks to months after the intervention provided that this afterload reduction was sustained. On the other hand, acute reductions in wall stress could also act to reduce subendocardial ischemia, which has been hypothesized to contribute to contractile dysfunction in many forms of cardiomyopathy.

One additional effect that has been observed after mass reduction surgery is a decrease in pulmonary capillary wedge pressure. However, a decrease in left ventricular filling pressure was not predicted by the present analysis. It is important to note that the present analysis simulated the effects of the mass reduction in the absence of other confounding factors that may be associated with this surgical procedure. First, mitral annuloplasty is often performed in concert with the mass reduction because of acutely altered geometry on mitral valve function along with the high incidence of significant mitral regurgitation in this patient population. Reduced regurgitant volume will decrease pulmonary wedge pressures, as well as contribute to an increase in forward stroke volume. It is important that the contribution of mitral annuloplasty to the potential clinical benefits observed after this procedure be clarified. Second, perioperative fluid management (including during cardiopulmonary bypass) and vasodilatory effects of anesthetics and other vasoactive

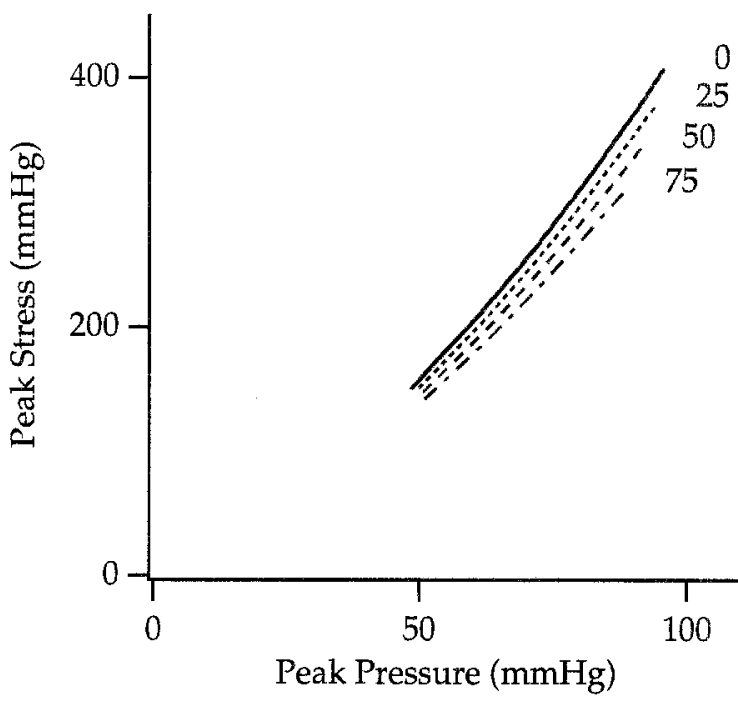

Fig. 7. Peak stress is shown as a function of peak chamber pressure at each of the four levels of mass reduction. These relations shift downward in response to mass reduction. The implication is that stress is reduced at a given peak chamber as a result of mass reduction.

agents used in the perioperative period (e.g., nitrates, $\beta$-agonists, phosphodiesterase inhibitors) will also have a profound impact on pulmonary wedge pressure. With these factors in mind, as pointed out previously, it is important to note that because multiple factors can influence pulmonary capillary wedge pressure, a decrease in this parameter does not necessarily indicate an improvement in heart function. ${ }^{2}$

The results presented in this study have been derived by means of an analytic approach. Accordingly, the validity of the interpretations depend on the validity of the assumptions underlying the analysis. It is unlikely that the myocardium is truly homogeneous in terms of thickness and function, and therefore the resulting properties of the nonexcised portion may not simply relate to the volume of the compartment excised, as was assumed. In addition to assumptions detailed in the Methods and materials section, we assumed that there was no alteration in myocardial function caused by the presence of a suture line. However, most of the basic aspects have been tested and validated in multiple experimental models. ${ }^{7}$ Also, as noted earlier, similar analytic approaches have been used successfully in the past by one group of investigators to study other complex hemodynamic phenomena involving interactions between the heart and vasculature..$^{2-4}$ Addi- 
tionally, results of our finite element analysis reveal the same changes in ESPVR and EDPVR as were found with the multicompartment model; concordance between these two different methods supports the validity of the results. Nevertheless, the results should be viewed as providing qualitative insights that are subject to verification. It is not the intention of such an analysis to provide detailed quantitative predictions of clinical scenarios, because each patient profile will vary significantly. The utility of this type of analysis is clear, however, particularly when so little detailed information is available from either experimental or clinical settings. Also, it will be a challenge to create an appropriate animal model with which to obtain such information. The rapid cardiac pacing model used in dogs and pigs has the disadvantage that a significant amount of function recovers once pacing is stopped. The coronary microembolization model might be useful, but the degree of ventricular dysfunction seen in that model (with ejection fractions typically about $30 \%$ ) does not approach the degree of dysfunction seen in patients who are receiving this therapy (with ejection fractions well below $20 \%$ ). ${ }^{16}$

In summary, the results of the present analysis reveal that improvements in ejection fraction, endsystolic elastance, and preload recruitable stroke work after ventricular mass reduction surgery have different implications than when such changes are observed with inotropy resulting from acute pharmacologic therapy. This difference stems predominantly from the fact that mass reduction will cause changes in diastolic ventricular properties, and consequently overall pump function (the Frank-Starling relationship) does not improve as would be observed with a purely inotropic intervention. On the other hand, the geometric rearrangement leads to a reduction in wall stress for a given level of pressure generation, thus implying an increase in the efficiency with which wall stress is transduced into intraventricular pressure. Whether this may translate into long-term benefits depends, in part, on the degree of afterload reduction that is maintained and in part on whether the underlying pathophysiologic process that led to the cardiomyopathy in the first place continues to progress. Finally, several other incidental factors associated with the procedure may contribute to clinical benefits of ventricular mass reduction (e.g., concomitant mitral annuloplasty, perioperative pharmacologic and fluid management). Results of ongoing clinical trials will establish whether this procedure truly offers sustained benefit to patients with end-stage cardiomyopathy; however, it will be equally important to study the underlying physiology of this procedure. Such understanding may ultimately contribute to devising appropriate patient selection criteria, tailoring the surgical procedure (e.g., how much muscle should be removed), and helping guide future research efforts aimed at optimizing the effects of this novel form of therapy. In this regard, the results of the present analysis provide a strong foundation for such efforts.

\section{REFERENCES}

1. Altman L. Brazil surgeon develops a bold, promising operation for patients with heart failure. New York Times 1996 June 14, 1996:A16.

2. Burkhoff D, Tyberg JV. Why does pulmonary venous pressure rise following the onset of left ventricular dysfunction? Results of a theoretical analysis of acute heart failure. Am J Physiol 1993;265:H1819-28.

3. Santamore WP, Burkhoff D. Hemodynamic consequences of ventricular interaction as assessed by model analysis. Am $\mathbf{J}$ Physiol 1991;260:H146-57.

4. Dickstein ML, Burkhoff D. A theoretical analysis of the effect of pulmonary vasodilation on pulmonary venous pressure: implications for inhaled nitric oxide therapy. J Heart Lung Transplant 1996;15:715-21.

5. Suga $H$, Sagawa K. Instantaneous pressure-volume relationships and their ratio in the excised, supported canine left ventricle. Circ Res 1974:35:117-26.

6. Arts T, Bovendeerd PHM, Prinzen FW, Reneman RS. Relation between left ventricular cavity pressure and volume and systolic fiber stress and strain in the wall. Biophys J 1991;59:93-102.

7. Sagawa K, Maughan WL, Suga $\mathbf{H}$, Sunagawa K. Cardiac contraction and the pressure-volume relationship. New York: Oxford University Press, 1988:480.

8. Sunagawa K, Maughan W, Sagawa K. Effect of regional ischemia on the left ventricular end-systolic pressure-volume relationship of isolated canine hearts. Circ Res 1983;52: 170-8.

9. Park RC, Little WC, O'Rourke RA. Effect of alteration of left ventricular activation sequence on the left ventricular end-systolic pressure-volume relation in close-chest dogs. Circ Res 1985;57:706-17.

10. Burkhoff D, Oikawa RY, Sagawa K. Influence of pacing site on canine left ventricular contraction. Am J Physiol 1986;251: H428-35.

11. Maughn WL, Sunagawa K, Sagawa K. Ventricular systolic interdependence: volume elastance model in isolated canine hearts. Am J Physiol 1987;253:H1381-90.

12. Rubin SA, Fishbein MC, Swan HJ. Compensatory hypertrophy in the heart after myocardial infarction in the rat. $\mathbf{J}$ Am Coll Cardiol 1983;1:435-41.

13. Strobeck EJ, Sonnenblick EH. Pathophysiology of heart failure. In: Levine HJ, Gaasch WH, editors. The ventricle: basic and clinical aspects. Boston: Martinus Nijhoff, 1985: 209-24.

14. Schwartz K, Boheler KR, de la Bastie D, Lompre AM, Mercadier JJ. Switches in cardiac muscle gene expression as 
a result of pressure and volume overload. Am J Physio 1992;262:R364-9.

15. Morgan HE, Baker KM. Cardiac hypertrophy: mechanical, neural and endocrine dependence. Circulation 1991;83:1325.

16. Todaka K, Leibowitz D, Homma S, Stennett RA, Packer M, Burkhoff D. Characterizing ventricular mechanics and energetics following repeated coronary microembolization. Am J Physiol. In press.

\section{Appendix}

Relation between mass removed and chamber volume. The LV was modeled as rotationally symmetric, hemielliptic chamber, with short-axis radius at the base $R_{S}$, long-axis length of $\mathrm{L}$ (the length from the base to the apex), and uniform wall thickness $T_{w}$. Heart reduction was assumed to remove a wedge-shaped portion of myocardium such that $R_{S}$ decreased while leaving starting $T_{w}$ and $L$ unchanged. The value for $L$ was $9 \mathrm{~cm}$, and the starting values for $T_{W}$ and $R_{S}$ were $1.5 \mathrm{~cm}$ and $3.8 \mathrm{~cm}$, respectively. LV chamber volume $\left(\mathrm{V}_{\mathrm{LV}}\right)$ was calculated as:

$$
\mathrm{V}_{\mathrm{LV}}=0.5 \times 4 / 3 \pi \mathrm{L}\left(\mathrm{R}_{\mathrm{S}}\right)^{2}
$$

For the baseline condition (before mass reduction, $\mathrm{R}_{\mathrm{S}}=$ $3.8 \mathrm{~cm}$ ); $V_{\mathrm{LV}}$ therefore equaled $270 \mathrm{ml}$. As $\mathrm{R}_{\mathrm{S}}$ was decreased, the chamber volume decreased in a nonlinear fashion. The coefficient of chamber volume reduction (r) is calculated by means of the formula:

$$
\mathrm{r}=\left(270-\mathrm{V}_{\mathrm{LV}}\right) / 270
$$

$\mathrm{LV}$ wall volume $\left(\mathrm{V}_{\mathrm{W}}\right)$ was calculated according to the following equation:

$$
\mathrm{V}_{\mathrm{W}}=\left(0.5 \times 4 / 3 \pi\left[\mathrm{L}+\mathrm{T}_{\mathrm{W}}\right]\left[\mathrm{R}_{\mathrm{S}}+\mathrm{T}_{\mathrm{W}}\right]^{2}\right)-\mathrm{V}_{\mathrm{LV}}
$$

$\mathrm{LV}$ wall mass $\left(\mathrm{M}_{\mathrm{LV}}\right)$ was calculated as the product of myocardial wall density $(0.96)$ and $V_{W}$. The mass excised $\left(M_{X}\right)$ varied with the reduction in $R_{S}$ and was calculated by combining equations 7 and 9 and solving numerically.

Cardiovascular simulation. The set of equations used to simulate the entire cardiovascular system has been fully described in previous publications. ${ }^{2-4}$ The RV and LV were coupled to pulmonary and systemic arterial and venous vascular beds; these beds were composed of a proximal characteristic resistance, an arterial resistance, a venous resistance, and venous and arterial compliances. The value of each parameter of the model (Appendix Table I) was set to be appropriate for a $75 \mathrm{~kg}$ man with dilated cardiomyopathy. Stressed blood volume was initially set at $3000 \mathrm{ml}$ and was reduced in increments of 250 $\mathrm{ml}$ to vary preload.

Finite element analysis. The purpose of this finite element analysis is to provide a second and independent analytic method of determining the effect of mass reduction on pressure-volume relations. ESPVRs and EDPVRs were determined assuming the same hemielliptic model described earlier. At each level of mass reduction, the relationship between $R_{S}$ and $T_{W}$ was calculated from
Appendix Table I. Parameter values for vasculature, right and left ventricles, which were

\begin{tabular}{|c|c|c|}
\hline Vascular parameters & Pulmonary & Systemic \\
\hline Arterial resistance (Wood units) & 1.0 & 15.5 \\
\hline Characteristic resistance (Wood units) & 0.3 & 0.3 \\
\hline Venous resistance (Wood units) & 0.25 & 0.25 \\
\hline Arterial capacitance $\left(\mathrm{ml} \cdot \mathrm{mm} \mathrm{Hg}^{-1}\right)$ & 13 & 1.3 \\
\hline Venous capacitance $\left(\mathrm{ml} \cdot \mathrm{mm} \mathrm{Hg}^{-1}\right)$ & 8 & 150 \\
\hline Heart function parameters & $R V$ & $L V$ \\
\hline Maximum elastance $\left(\mathrm{mm} \mathrm{Hg} \cdot \mathrm{ml}^{-1}\right)$ & 0.3 & 0.5 \\
\hline Unstressed volume (ml) & 10 & 50 \\
\hline Time to end-systole (msec) & 175 & 175 \\
\hline Time constant of relaxation (msec) & 25 & 25 \\
\hline Scaling factor for EDPVR (mm $\mathrm{Hg}$ ) & 0.13 & 0.16 \\
\hline Exponent for $\operatorname{EDPVR}\left(\mathrm{K}, \mathrm{ml}^{-1}\right)$ & 0.019 & 0.024 \\
\hline Heart rate (beats/min) & 75 & 75 \\
\hline
\end{tabular}
chosen to be appropriate for a $70 \mathrm{~kg}$ man with dilated cardiomyopathy

equation 11. The midwall circumference $\left(\mathrm{C}_{\mathrm{mw}}\right)$ is then calculated as

$$
\mathrm{C}_{\mathrm{muw}}=0.5 \times 4 / 3 \pi \mathrm{L}\left(\mathrm{R}_{\mathrm{S}}+0.5 \mathrm{~T}_{\mathrm{W}}\right)^{2}
$$

Midwall strain $(\epsilon)$ at the base is given by

$$
\epsilon=\left(\mathrm{C}_{\mathrm{mw}}-\mathrm{C}_{\mathrm{mw}} \mathrm{o}\right) /\left(\mathrm{C}_{\mathrm{mw}} \max -\mathrm{C}_{\mathrm{mw}} \mathrm{o}\right)
$$

where $\mathrm{C}_{\mathrm{mw}} \mathrm{O}$ is the midwall circumference when chamber volume equals $V_{O}$, and $C_{m w}$ max is the circumference corresponding to the starting chamber volume. Average stress $(\sigma)$ is then calculated according to empiric functions for systole and diastole:

$$
\begin{gathered}
\sigma=160 \epsilon^{2.5} \text { at end-systole } \\
\sigma=60 \epsilon^{7} \text { at end-diastole }
\end{gathered}
$$

The particular parameters for systolic and diastolic stress were chosen to approximate the ESPVR and EDPVR used in the multicompartment model and represent the elemental myocardial properties used in this analysis. LV pressure $\left(\mathrm{P}_{\mathrm{LV}}\right)$ is calculated as

$$
\mathrm{P}_{\mathrm{LV}}=\mathrm{A}_{\mathrm{w}} \sigma / \mathrm{A}_{\mathrm{ch}}
$$

where $A_{w}$, the long-axis myocardial cross-sectional area, is given by

$$
\mathrm{A}_{\mathrm{iw}}=0.5 \pi\left(\left[\mathrm{R}_{\mathrm{S}}+\mathrm{T}_{\mathrm{W}}\right]\left[\mathrm{L}+\mathrm{T}_{\mathrm{W}}\right]-\mathrm{R}_{\mathrm{S}} \mathrm{L}\right)
$$

and $\mathrm{A}_{\mathrm{ch}}$, the long-axis chamber cross-sectional area, is given by

$$
\mathrm{A}_{\mathrm{ch}}=0.5 \pi \mathrm{R}_{\mathrm{S}} \mathrm{L}
$$

Because LV volume (equation 9) and LV pressure (equation 16) are both functions of $R_{S}$, pressurevolume relationships are thus calculated over a range of 


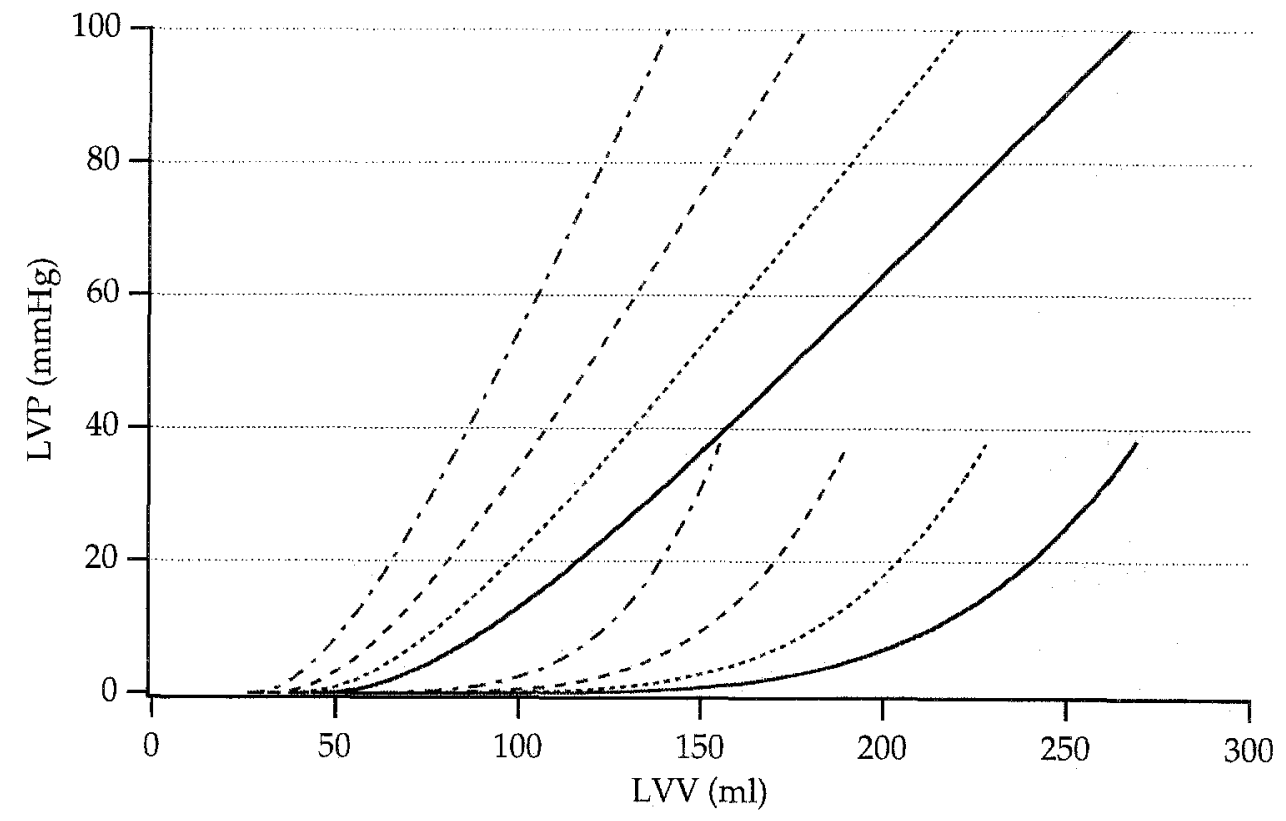

Appendix Fig. 1. Left ventricular pressure-volume relations determined from finite element analysis at each of the four levels of mass reduction $(0 \mathrm{gm}$, solid line; $25 \mathrm{gm}$, dotted line; $50 \mathrm{gm}$, dashed line; $75 \mathrm{gm}$, dotted/dashed line). As mass is reduced from 0 to $75 \mathrm{gm}$, the ESPVR slope and curvature of the EDPVR both increase; these changes are similar to that shown in Fig. 2 derived from compartmental modeling.

chamber volumes by varying $R_{\mathbf{s}}$. Heart reduction was assumed to reduce myocardial mass, while leaving myocardial stress-strain relations (equations 14 and 15) and starting $\mathrm{T}_{\mathrm{W}}$ unchanged. ESPVRs and EDPVRs thus determined are illustrated in Appendix Fig. 1. Similar to the multicompartment model, this finite element analysis shows that mass reduction causes equivalent shifts in the ESPVRs and EDPVRs.

\section{Commentary}

Although the ventricular reduction operation for dilated cardiomyopathy has received tremendous attention in the lay press and in a few medical publications, the physiologic basis of this procedure has not been completely established either in the laboratory or in clinical settings. In particular, the relative importance of mitral valve repair versus ventricular resection is unclear. The more practical issue of how much myocardium to remove is addressed in the article by Dickstein and associates. The authors used a multiple compartment elastance model to demonstrate that increasing removal of up to $75 \mathrm{gm}$ of myocardium caused progressive increase in left ventricular contractility (measured by end-systolic elastance), increase in left ventricular ejection fraction, and decrease in left ventricular enddiastolic volume. These improvements were at the expense of decreased stroke volume, decreased ventricular diastolic compliance, and a small decrease in left ventricular systolic pressure. Thus, as with left ventricular aneurysmectomy, excessive ventricular resection can result in patient morbidity and even death because of the inability to maintain an acceptable cardiac output without excessive pulmonary venous pressure or excessive heart rate.

Dickstein and associates provide a foundation to address two remaining questions facing the surgeon who considers a ventricular reduction operation.

1. How can it be demonstrated whether a given patient has sufficient intrinsic myocardial contractility to benefit from ventricular reduction?

2. What is the optimal volume of myocardium to resect in a given patient to obtain maximal benefit without excessive reduction in stroke volume or excessive increase in pulmonary venous pressure?

Answer to these questions will await quantitative work like that of Dickstein and associates, both in the animal laboratory and in thoughtful clinical studies.

Donald D. Glower, $M D$ Associate Professor of Surgery Duke University Medical Center Durham, NC 27710 $12 / 1 / 81394$ 